\title{
Properties of Anti-Adjacency Matrix of Cyclic Directed Windmill Graph $\mathrm{K} \overrightarrow{(4, \mathrm{n})}$
}

\author{
Muhammad Sabili Robbi Solihin*, S Aminah, Suarsi Utama \\ Department of Mathematics, Faculty of Mathematics and Natural Sciences, Universitas Indonesia, INDONESIA. \\ * robbisolihin@sci.ui.ac.id
}

\begin{abstract}
Anti-adjacency matrix is a way to represent a directed graph as a square matrix, whose entries show whether there is a directed edge from a vertex to another one. This paper focuses on the properties of anti-adjacency matrix of windmill graph $\vec{K}(4, n)$, such as its characteristic polynomial and eigenvalues. The general form of characteristic polynomial is established by analyzing the degree of vertices and edges, and the cyclic induced subgraphs. Furthermore, the eigenvalues of a windmill graph $\vec{K}(4, n)$ and its multiplicity are derived from the general form of its characteristic polynomial.
\end{abstract}

Keywords- Graph; Anti-adjacency matrix; Characteristic polynomial; Eigenvalue.

\section{INTRODUCTION}

Let $V(G)=\left\{v_{1}, v_{2}, \ldots, v_{n}\right\}$ is a non-empty set of vertices of a directed graph $G$, then the adjacency matrix of the graph $G$ is a $n \times n$ matrix $A(G)=\left[a_{i j}\right]$, where $a_{i j}$ is equal to 1 if there exists a directed edge from $v_{i}$ to $v_{j}$ and equal to 0 elsewhere [2]. Anti-adjacency matrix is an opposite of an adjacency matrix. Anti-adjacency matrix of a directed graph is always a square matrix, so we can find its characteristics equation and eigenvalues of that matrix.

In this article, we define a class of graph named windmill graph, denoted with $\overrightarrow{\mathbb{K}}(m, n)$ s which consists of $n$ cycle graph $C_{m}$ that share one common vertex. This class of graph is a generalization of an already familiar class of graph called friendship graph, which properties are already discussed [3][4]. A friendship graph is isomorphic to the windmill graph $\overrightarrow{\mathbb{K}}(3, n)$. In this article, we focus on $\overrightarrow{\mathbb{K}}(4, n)$ windmill graph, where we find a general form of its characteristics equation for $=1,2,3, \ldots$. We can use the general form to derive some of its eigenvalues and multiplicity. In the study of graph, eigenvalues could be used to describe the topological structure of a graph, and to determine many intrinsic structures of a graph, such as spanning trees, Hamiltonian path, induced subgraphs, etc.

\section{PRELIMINARIES}

Anti-adjacency matrix of graph $G$ was a matrix $B(G)=J-A(G)$ where $A(G)$ was an adjacency matrix of a graph $G$ and $I$ was a matrix whose all entries were 1 and its size was the same with $A(G)$ [2]. Afterwards, what we meant by "the determinant of graph $G$ " and "the characteristics equation of graph $G$ " was the determinant of the anti-adjacency matrix of graph $G$ and the characteristics equation of the antiadjacency matrix of graph $G$, except if stated differently. We mainly used the theorem taken from Wildan [1].

Windmill $\operatorname{graph}(\boldsymbol{m}, \boldsymbol{n})$ is a graph that formed from $n$ cycle graphs $C_{m}$ having 1 common vertex. Afterwards, this graph is denoted with $\overrightarrow{\mathrm{K}}(m, n)$. We can call the subgraph in the form of cycle graph $C_{m}$ as "windmill leaf". This article only focuses on $\overrightarrow{\mathrm{K}}(4, n)$ graph.

The set of in neighbourhood of a vertex $v$ from a directed graph $G$ is a set of all vertices that have some edges going into $v_{v}$ denoted with $\mathrm{N}^{+}(v)$, while the set of out neighbourhood of a vertex $v$ from a directed graph $G$ is a set of all vertices that have some edges going out from $v$, denoted with $\mathrm{N}^{-}(v)$. The determinant of a graph with two vertices that have the same set of in neighbourhood or out neighbourhood is equals to 0 because the anti-adjacency matrix will have two columns or two rows that have exactly the same entries. [1]

\section{Lemma 1}

Let a directed graph $G$ have two vertices $u$ and $\mathrm{v}$, where $u \neq v, \quad$ that satisfied $N^{+}(v)=N^{+}(u)$ $\operatorname{or} N^{-}(v)=N^{-}(u)$, then $\operatorname{det}(B(G))=0$.

Some special graphs taken from Wildan [1] will be used to determine the coefficients of the characteristics equation of a graph

\section{MAIN RESULT}

The characteristics equation of a graph had a form $\lambda^{m}+b_{1} \lambda^{m-1}+b_{2} \lambda^{m-2}+\cdots+b_{m-1} \lambda+b_{m}=0$,

where $\boldsymbol{m}$ was the number of vertices, so we could find the general form of characteristics polynomial of $\overrightarrow{\mathbf{K}}(4, \boldsymbol{n})$ by finding all value of $\boldsymbol{b}_{1}, \boldsymbol{b}_{2}, \ldots, \boldsymbol{b}_{m}$ in respect of $\boldsymbol{n}$.

By using mathematical induction we proved that the order of vertices and edges of graph $\overrightarrow{\mathbf{K}}(4, \boldsymbol{n})$ were $\mathbf{3 n}+\mathbf{1}$ and 4n. The value of $b_{1}$ was equal to negative value of the number of vertices on the graph and the value of $\boldsymbol{b}_{2}$ was equal to the number of edges on the graph [1]. We combined this facts into Theorem 2 . 


\section{Theorem 2}

Given a graph $\overrightarrow{\mathbf{K}}(4, \mathbf{n})$ where $\mathbf{V}(\mathbf{K}(\mathbf{4}, \mathbf{n}))=\{\mathbf{1}, \mathbf{2}, \ldots, \mathbf{m}\}$, with $\mathbf{m}=\|\mathbf{V}\|=\mathbf{3 n}+\mathbf{1}$ Let $\lambda^{m}+b_{1} \lambda^{m-1}+b_{2} \lambda^{m-2}+\cdots+b_{m-1} \lambda+b_{m}=0$ was the characteristics equation of the anti-adjacency matrix of graph $\overrightarrow{\mathbf{K}}(4, \mathbf{n})$, then $\mathbf{b}_{1}=-(3 \mathbf{n}+\mathbf{1})$ and $\mathbf{b}_{2}=\mathbf{4 n}$.

\section{Theorem 3}

Given graph $\overrightarrow{\mathbf{K}}(4, \mathbf{n})$ where $\mathbf{V}(\mathbf{K}(\mathbf{4}, \mathbf{n}))=\{\mathbf{1}, \mathbf{2}, \ldots, \mathbf{m}\}$, with $\mathbf{m}=\mid \mathbf{V} \|=\mathbf{3 n}+\mathbf{1}$.

$\lambda^{m}+b_{1} \lambda^{m-1}+b_{2} \lambda^{m-2}+\cdots+b_{m-1} \lambda+b_{m}=0$ was the characteristics equation of the anti-adjacency matrix of graph $\overrightarrow{\mathbf{K}}(4, \mathbf{n})$, then $\boldsymbol{b}_{3}=-\left(\boldsymbol{n}^{2}+\mathbf{3 n}\right)$.

\section{Proof:}

According to Wildan, the value of $\boldsymbol{b}_{3}$ was equal to - $\left(S_{3}+\mathbf{2} S_{\mathrm{T} 1.1(3)}\right)$, where $S_{3}$ was the number of the induced acyclic subgraphs with 3 vertices that had Hamiltonian path and $S_{\mathrm{T1.1(3)}}$ was the number of induced cyclic subgraph in the form of T1.1(3) [1]. We could easily see that graph $\overrightarrow{\mathbf{K}}(4, \boldsymbol{n})$ did not contain an induced cyclic subgraph in the form of T1.1(3), so the value of $\boldsymbol{b}_{3}$ was equal to $-\boldsymbol{S}_{3}$. We could prove that the number of induced acyclic subgraph with 3 vertices that had Hamiltonian path was equal to $\boldsymbol{n}^{2}+\mathbf{3}$, so we could conclude that $b_{3}=-S_{3}=-\left(n^{2}+3 n\right)$.

\section{Theorem 4}

Given graph $\overrightarrow{\mathbf{K}}(4, \mathbf{n})$ where $\mathbf{V}(\mathbf{K}(\mathbf{4}, \mathbf{n}))=\{\mathbf{1}, \mathbf{2}, \ldots, \mathbf{m}\}$, with

$$
\mathbf{m}=\|\mathbf{V}\|=\mathbf{3 n}+\mathbf{1} \text {. }
$$

$\lambda^{m}+b_{1} \lambda^{m-1}+b_{2} \lambda^{m-2}+\cdots+b_{m-1} \lambda+b_{m}=0$ was the characteristics equation of anti-adjacency matrix of graph $\overrightarrow{\mathbf{K}}(4, \mathbf{n})$, then $b_{4}=\mathbf{2} n^{2}+\boldsymbol{n}$.

\section{Proof:}

The value of $\boldsymbol{b}_{\mathbf{4}}$ depended on the number of induced acyclic subgraphs with 3 vertices that had Hamiltonian path, and the number of induced cyclic subgraphs in the form of T1.1(4), T1.2(4,0), T1.2(4,1), T2.1(4,0), T2.1(4,2), T2.1(4,3), $\mathrm{T} 2.2(4,2)$, and T2.2(4,3) [1]. From all the graphs mentioned, graph $\overrightarrow{\mathbf{K}}(4, \boldsymbol{n})$ only had subgraph in the form of T1.1(4), so $b_{4}=S_{4}+3 S_{T 1.1(4)}$, where $S_{T 1.1(4)}$ was the number of induced cyclic subgraph in the form of T1.1(4). We could prove that the number of $\boldsymbol{S}_{\mathbf{4}}+\mathbf{3} \boldsymbol{S}_{\boldsymbol{T 1 . 1 ( 4 )}}$ from graph $\overrightarrow{\mathbf{K}}(4, \boldsymbol{n})$ is $2 n^{2}+n, \quad$ so we could conclude that $b_{4}=-\left(s_{4}+3 S_{T 11(4)}\right)=-\left(2 n^{2}+n\right)$.

\section{Theorem 5}

Given graph $\overrightarrow{\mathbf{K}}(4, \mathbf{n})$ where $\mathbf{V}(\mathbf{K}(\mathbf{4}, \mathbf{n}))=\{\mathbf{1}, \mathbf{2}, \ldots, \mathbf{m}\}$, with $\quad \mathbf{m}=|\mathbf{V}|=\mathbf{3 n}+\mathbf{1}$ Let $\lambda^{m}+b_{1} \lambda^{m-1}+b_{2} \lambda^{m-2}+\cdots+b_{m-1} \lambda+b_{m}=0$ was the characteristics equation of the anti-adjacency matrix of graph $\overrightarrow{\mathbf{K}}(4, \mathbf{n})$, then $\boldsymbol{b}_{\mathbf{5}}=\mathbf{0}$.

\section{Proof:}

According to Theorem 1

$$
b_{5}=(-1)^{5}\left(\sum_{j_{1}=1}^{w_{1}}\left|B\left(\langle U\rangle_{\text {acyelic }}\right)_{5}^{\left[j_{1}\right\rangle}\right|+\sum_{j_{2}=1}^{W_{2}}\left|B\left(\langle U\rangle_{\text {cyelic }}\right)_{5}^{\left[j_{2}\right\rangle}\right|\right) \text {, }
$$

We would prove the acyclic part and cyclic part separately.

\section{Acyclic part}

On graph $\overrightarrow{\mathbf{K}}(4, \boldsymbol{n})$, there was only one induced acyclic subgraph that had Hamiltonian path, that was a subgraph that formed from two vertices from the windmill leaf that went into centre vertex, another two vertices from distinct windmill leaf that came from the centre vertex, and the centre vertex itself. From two distinct windmill leaf, we could get two distinct subgraphs, so for graph $\overrightarrow{\mathbf{K}}(4, \boldsymbol{n})$ that had $\boldsymbol{n}$ windmill leaf, there were $\mathbf{2} \boldsymbol{C}\left(\begin{array}{l}n \\ 2\end{array}\right)=\mathbf{2}\left(\frac{n !}{2(n-2)}\right)=\boldsymbol{n}(\boldsymbol{n}-\mathbf{1}) \quad$ subgraph. We knew that the determinant of acyclic graph that had Hamiltonian path was 1 , so we concluded that

$\sum_{i=1}^{w_{1}}\left|B\left(\langle U\rangle_{\text {acyclic }}\right)_{5}^{\left(j_{1}\right)}\right|=\sum_{j=1}^{n(n-1)} 1=n(n-1)$.

Cyclic part

In order to get a cyclic induced subgraph, there had to be at least one complete windmill leaf. There were three variants that could be formed, and only one variant had non-zero determinant. So, it was sufficient to only count the number of cyclic induced subgraphs in the form of that variant. That variant could be formed by choosing two distinct windmill leaves. So, the number of subgraphs in the form of G3 was $P\left(\begin{array}{l}n \\ 2\end{array}\right)=\left(\frac{n !}{(n-2) !}\right)=n(n-1)$. So we get

$\sum_{f_{2}=1}^{w_{2}}\left|B\left(\langle U\rangle_{\text {cyelic }}\right)_{5}^{\left.\mathbb{f}_{2}\right]}\right|=\sum_{f_{2}=1}^{n(n-1)}(-1)=-(n(n-1))$.

Combining cyclic part with acyclic part, we got

$b_{5}=(-1)^{5}\left(\sum_{j_{1}=1}^{w_{1}}\left|B\left(\langle U\rangle_{a c y c l i i}\right)_{5}^{\left(j_{1}\right)}\right|+\sum_{j_{2}=1}^{w_{2}}\left|B\left(\langle U\rangle_{c y c l i c}\right)_{5}^{\left(j_{2}\right)}\right|\right)=n(n-1)-(n(n-1))=0$. .

To find the values of $b_{6,}, b_{7, \ldots,} b_{m,}$ we defined six graphs that served as the basic forms of cyclic induced subgraphs that could be formed from graph $\overrightarrow{\mathrm{K}}(4, n)$. Table 1 shows all six graphs and their notations.

Afterwards, all cyclic induced subgraph that formed from graph $\overrightarrow{\mathbb{K}}(4, n)$ were denoted as $A_{a} B_{b} C_{c} D_{d} E_{e} F_{f}$ with rules as follow:

- The set of indexes $\left\{a, b, c, d, e_{x} f\right\}$ in succession order represented the number of basic forms $A$ to $F$ that existed on a subgraph that formed from graph $\overrightarrow{\mathbb{K}}(4, n)$

- If there were some indexes with value of 1 , that particular indexes could be omitted. As an example, $A_{3} B_{1} C_{2} D_{1} E_{1} F_{4}$ could be written as $A_{3} B C_{2} D E F_{4}$ 
- If there were some indexes with value of 0 , the entire letters and indexes could be omitted. As an example,

\section{Lemma 2}

$A_{2} B_{0} C_{0} D_{0} E_{2} F$ could be written as $A_{2} E_{2} F$

Let $U=A_{a} B_{b} C_{c} D_{d} E_{e} F_{f}$ was a cyclic induced subgraph with $\operatorname{Max}\{a, b\} \geq 2$ or $\operatorname{Max}\left\{c, d, e_{,} f\right\} \geq 1$, then $\operatorname{det}(B(U))=0$.

TABLE I. BASIC FORM OF SUBGRAPHS THAT COULD BE FORMED FROM GRAPH $\overrightarrow{\mathrm{K}}(4, n)$

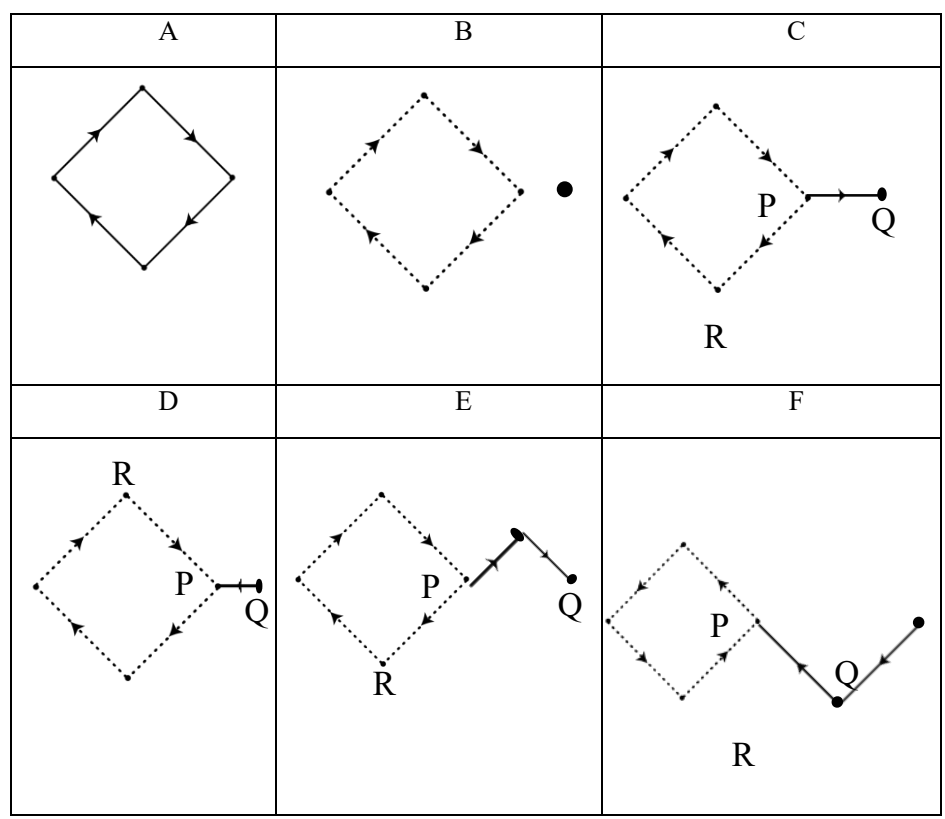

\section{Proof:}

- $\quad$ For $a \geq 2$, look at Figure 4

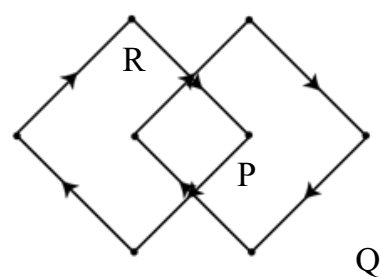

Figure 4: The cyclic induced subgraph $A_{a} B_{b} C_{c} D_{d} E_{e} F_{f}$ with $a \geq 2$

since $N^{-}(Q)=N^{-}(R)=\{P\}, \quad$ according to Lemma $1, \operatorname{det}(B(U))=0$

- $\quad$ For $b \geq 2$, look at Figure 5

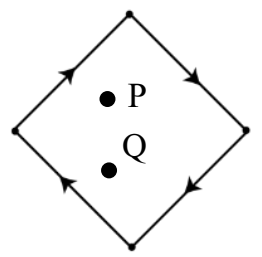

Figure 5: The cyclic induced subgraph $A_{a} B_{b} C_{c} D_{d} E_{e} F_{f}$ with $b \geq 2$

since $N^{+}(P)=N^{+}(Q)=\{\emptyset\}, \quad$ according to Lemma $1, \operatorname{det}(B(U))=0$
- $\quad$ For $c \geq 1$ and $e \geq 1$, look at Table 3 part $\mathrm{C}$ and $\mathrm{E}$ respectively, since $N^{+}(Q)=N^{+}(R)=\{P\}$, according to Lemma $1, \operatorname{det}(B(U))=0$

- $\quad$ For $d \geq 1$ and $f \geq 1$, look at Table 3 part D and $\mathrm{F}$ respectively, since $N^{-}(Q)=N^{-}(R)=\{P\}$, according to Lemma $1, \operatorname{det}(B(U))=0$.

From Lemma 2 we could conclude that all cyclic induced subgraphs that had at least 6 vertices had determinant equal to 0 .

\section{Theorem 6}

Given graph $\overrightarrow{\mathbb{K}}(4, \mathrm{n})$ where $V(K(4, n))=\{1,2, \ldots, \mathrm{m}\}$, with $=|\mathrm{V}|=3 \mathrm{n}+1$.

$\lambda^{m}+b_{1} \lambda^{m-1}+b_{2} \lambda^{m-2}+\cdots+b_{m-1} \lambda+b_{m}=0$ was the equation characteristics of the anti-adjacency matrix of graph $\overrightarrow{\mathbb{K}}(4, \mathrm{n})$, then $b_{i}=0$, For $i=6,7, \ldots, m$.

\section{Proof:}

- $\quad$ Acyclic part

The graph $\overrightarrow{\mathbb{K}}(4, n)$ did not contain acyclic induced subgraph with Hamiltonian path with number of vertices at least 5 , so

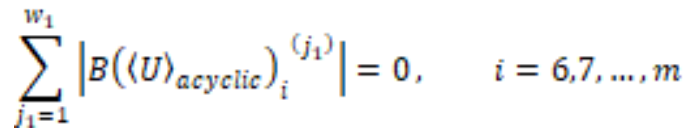

- $\quad$ Cyclic part

According to Lemma 2, all determinants of cyclic induced subgraph with number of vertices at least 6 would be equal to 0 , so

$$
\sum_{\delta_{2}=1}^{w_{2}}\left|B\left(\langle U\rangle_{\text {cyclic }}\right)_{i}^{\left[f_{2}\right)}\right|=0, \quad i=6,7, \ldots, m
$$

According to Theorem 1, we concluded that

$$
b_{i}=(-1)^{i}\left(\sum_{j_{1}=1}^{W_{1}}\left|B\left(\langle U\rangle_{\text {acyclic }}\right)_{i}^{\left(j_{1}\right)}\right|+\sum_{j_{2}=1}^{W_{2}}\left|B\left(\langle U\rangle_{c y c l i c}\right)_{i}^{\left(j j_{2}\right\rangle}\right|\right)=0, \quad i=6,7_{, \ldots}, m_{4} \text {. }
$$

\section{Theorem 7}

Let $\mathrm{B}$ the anti-adjacency matrix of graph $\overrightarrow{\mathrm{K}}(4, n)$, then one of the eigenvalue of $B$ was $\lambda=0$, with multiplicity $m(\lambda)=3 n-3$.

\section{Proof:}

According to Theorem 2, Theorem 3, Theorem 4, Theorem 5 , and Theorem 6 , given graph $\overrightarrow{\mathrm{K}}(4, n)$ with $V(\mathrm{~K}(4, n))=\{1,2, \ldots, \mathrm{m}\}$, where $m=|V|=3 n+1$, the general form of the characteristics equation of graph $\overrightarrow{\mathbb{K}}(4, n)$ was

$$
\lambda^{m}-(3 n+1) \lambda^{m-1}+(4 n) \lambda^{m-2}-\left(n^{2}+3 n\right) \lambda^{m-3}+\left(2 n^{2}+n\right) \lambda^{m-4}=0
$$

Factorize $\lambda^{m-4}$ from all terms to get

$$
\lambda^{3-4}\left(\lambda^{4}-(3 n+1) \lambda^{3}+(4 n) \lambda^{2}-\left(n^{2}+3 n\right) \lambda+\left(2 n^{2}+n\right)\right)=0
$$


We easily saw that $\lambda=0$ was a root from that polynomial equation, so $\lambda=0$ was one of the eigenvalue of matrix $B$ with multiplicity $m(\lambda)=m-4=3 n+1-4=3 n-3$.

\section{CONCLUSION}

According to $\quad$ section
$\lambda^{m}+b_{1} \lambda^{m-1}+b_{2} \lambda^{m-2}+\cdots+b_{m-1} \lambda+b_{m}=0$ , was a characteristics equation for anti-adjacency matrix of a graph $\overrightarrow{\mathrm{K}}(4, n)$. Table 2 shows the value of its coefficient in respect to $n$.

TABLE II. COEFFICIENTS OF CHARACTERISTICS EQUATION OF ANTI-ADJACENCY MATRIX OF GRAPH $\overrightarrow{\mathbb{K}}(4, n)$

\begin{tabular}{|c|c|}
\hline Coefficient Index & Coefficient Value \\
\hline$b_{1}$ & $-(3 n+1)$ \\
\hline$b_{2}$ & $4 n$ \\
\hline$b_{3}$ & $-\left(n^{2}+3 n\right)$ \\
\hline$b_{4}$ & $2 n^{2}+n$ \\
\hline$b_{5,}, b_{6, \ldots,}, b_{m}$ & 0 \\
\hline
\end{tabular}

We also get that one of its eigen value is 0 with multiplicitym $(\lambda)=n-3$.

\section{ACKNOWLEDGMENT}

This research is funded by Hibah PITTA UI [2307/UN2.R3.1/HKP.05.00/2018].

\section{REFERENCES}

[1] Wildan, Polinomial Karakteristik Anti-adjacency matrix dan Adjacency dari Graph Berarah Yang Diberi Orientasi, Depok: Universitas Indonesia, 2015, p, 41, p. 32, p. 56,

[2] R. B. Bapat, in Graphs, and Matrices, Springer, Berlin, 2010, p 25, p 37

[3] A. Abdollahi and S. H. Janbaz, Connected Graphs Cospectral With A Friendship Graphs, University of Isfahan, 2014

[4] S. Alikhani, J. I. Brown, S. Jahari, On the Domination Polynomials of Friendship Graphs, Yazd University, 2016

[5] R. S. Sheffield and L. McIlvenny, "Design and implementation of scientific inquiry using technology in a teacher education program", Int. J. Innov. Sci. Math. Educ., vol. 22, pp. 46-60, 2014. 